\title{
INVESTIGATING METERING CONVERTER OF CAPACITANCE USING ARDUINO PLATFORM
}

\author{
Aleksandr Vostrukhin ${ }^{1}$, Elena Vakhtina ${ }^{2}$ \\ ${ }^{1}$ Stavropol Technological Institute of Service, Russia; ${ }^{2}$ Stavropol State Agrarian University, Russia \\ avostrukhin@yandex.ru, eavakhtina@yandex.ru
}

\begin{abstract}
The task of improving electronic devices for measuring the moisture content of agricultural seeds is always relevant. Knowledge of the best ways, methods and technical solutions in a particular field of activity is the basis for creating innovative devices. However, manufacturers do not disclose the hardware and software of their devices. This circumstance does not allow performing a comparative analysis and choosing the best solution, which slows down the technical progress and engineering education to a certain extent. This paper discusses the hardware and the operation algorithm for the measuring converter of capacitance (MCC), which is designed for constructing a dielcometric USB-moisture meter for seeds of agricultural crops, as well as the study results of its main characteristics. Arduino hardware and software (IDE) were used. A diagram of an experimental MCC sample, a model of a measuring circuit in the Multisim program and recommendations on the selection of elements for reference and measuring RC-circuits are presented. Studies have shown that MCC can be used for building dielcometric USB-moisture meters of seeds based on microcontrollers containing: an analog comparator, a twochannel 8-bit pulse width a modulator (PWM), a 10-bit PWM, and a USB interface. The transients used in MCC are fundamental, so the research results can be used in engineering education to study embedded computing systems. Transient processes in RC-circuits can be simulated in the Multisim program, as well as investigated using the Arduino platform, which allows to observe the results of the conversions on a computer monitor.
\end{abstract}

Keywords: analog comparator, microcontroller, pulse width modulator, RC-circuit, sensor.

\section{Introduction}

The purpose of the article is to present the study results of the measuring converter of capacitance (MCC) into a binary code, which is proposed for constructing a dielcometric USB-moisture meter for seeds of agricultural crops.

The sensitivity of the MCC decreases with increasing capacitance. The dielectric constant of seeds increases with raising their moisture according to the exponential function law $[1 ; 2]$. However, in microprocessor-based moisture meters, calibration tables are used, therefore, the nonlinearity of the conversion characteristic does not affect the measurement accuracy. In this paper, we consider MCC, for which patent for an invention [3] and a certificate for state registration of the program have been obtained in Russia.

The circuit model of the proposed MCC is made in the Multisim program as shown in Fig. 1. The circuit contains: reference resistors $R_{o}$ and $R_{r}$; capacitive sensor $C_{s}$; reference capacitor $C_{r}$; generators XFG1 and XFG2 of rectangular pulses, allowing to control the duty cycle of the generated pulses; twochannel oscilloscope XSC1.

Oscillograms of voltages across the capacitive sensor $C_{s}=500 \mathrm{pF}$ (channel A) and the reference capacitor $C_{r}=250 \mathrm{pF}$ (channel B) are shown in Fig. 2. The frequency of rectangular pulses generated by the XFG1 and XFG2 generators is $100 \mathrm{kHz}, R_{o}=R_{r}=10 \mathrm{k} \Omega$.

As a result of modeling the circuit in Fig. 1, the dependence of the difference between the voltage amplitudes of the reference capacitor $C_{r}$ and the sensor $C_{s}$ on the capacitance of the last was obtained. This dependence is represented by equation (1):

$$
V_{r s}=V_{r}-V_{s},
$$

where $V_{r}$ and $V_{s}$ - voltage amplitudes across the reference capacitor $C_{r}$ and the sensor $C_{s}, \mathrm{~V}$.

The amplitude $V_{r}$ is determined by equation (2):

$$
V_{r}=V_{r \max }-V_{r \min },
$$

where $V_{r \max }$ and $V_{r \min }-$ maximum and minimum voltage across the reference capacitor $C_{r}, \mathrm{~V}$.

$V_{s}$ amplitude is determined similarly to (2):

$$
V_{s}=V_{s \max }-V_{s \min },
$$


where $V_{s \max }$ and $V_{s \min }-$ maximum and minimum voltage across the sensor $C_{s}, \mathrm{~V}$.

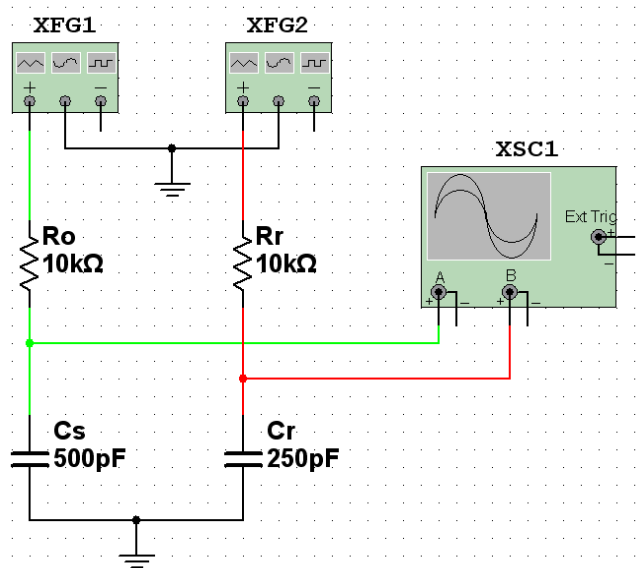

Fig. 1. Circuit model in Multisim for MCC research

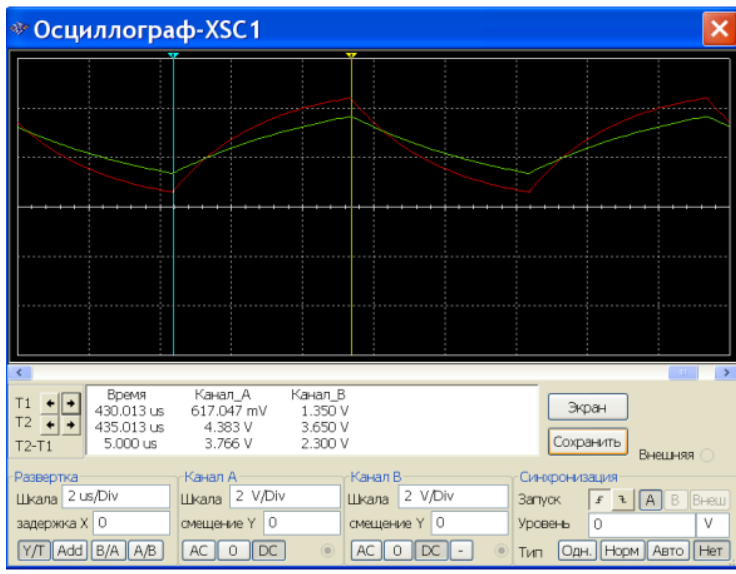

Fig. 2. Oscillograms of voltages across capacitors Cs (channel A) and Cr (channel B)

Table 1 shows the results of modeling the circuit in Fig. 1 and the data calculated using expressions (1), (2) and (3).

Results of circuit simulation in Multisim

Table 1

\begin{tabular}{|l|c|c|c|c|c|c|}
\hline $\mathbf{C s}, \mathbf{p F}$ & 250 & 300 & 350 & 400 & 450 & 500 \\
\hline $\boldsymbol{V}_{\boldsymbol{s} \min }, \mathbf{V}$ & 0.599 & 0.800 & 0.967 & 1.115 & 1.240 & 1.347 \\
\hline $\boldsymbol{V}_{\boldsymbol{s} \max }, \mathbf{V}$ & 4.402 & 4.206 & 4.029 & 3.886 & 3.762 & 3.654 \\
\hline $\boldsymbol{V}_{\boldsymbol{s}}=\boldsymbol{V}_{\boldsymbol{s m a x}}-\boldsymbol{V}_{\boldsymbol{s m i n}}, \mathbf{V}$ & 3.802 & 3.405 & 3.062 & 2.772 & 2.522 & 2.308 \\
\hline $\boldsymbol{V}_{\boldsymbol{r}}=\boldsymbol{V}_{\boldsymbol{r}}-\boldsymbol{V}_{\boldsymbol{s}}, \mathbf{V}$ & 0 & 0.39 & 0.74 & 1.03 & 1.28 & 1.49 \\
\hline
\end{tabular}

When the capacitance changes from $C_{s \min }=250 \mathrm{pF}$ to $C_{s \max }=500 \mathrm{pF}$, the amplitude changes from $3.802 \mathrm{~V}$ to $2.308 \mathrm{~V}$. The average sensitivity of the circuit in the entire range of the capacitance $C_{s}$ variation is determined by equation (4):

$$
S=\frac{V_{s \max }-V_{s \min }}{C_{s \max }-C_{s \min }}=\frac{3.802-2.308}{500-250}=5.976 \approx 6 \frac{\mathrm{mV}}{p F},
$$

When the capacitance changes from $C_{s \min }=25 \mathrm{pF}$ to $C_{s \max }=50 \mathrm{pF}$ and $R_{o}=100 \mathrm{k} \Omega$, the amplitude changes in the same range as for the capacitance changing from 250 to $500 \mathrm{pF}$. In this case, in accordance with expression (4), the average sensitivity of the circuit is:

$$
S=\frac{3.802-2.308}{50-25}=59.76 \approx 60 \frac{\mathrm{mV}}{\mathrm{pF}} .
$$

Thus, the sensitivity of this circuit is relatively high, and it makes sense of a microcontroller-based implementation.

The problem lies in measuring the instantaneous values of $V_{\text {smax }}$ and $V_{s \sin }$. AVR microcontrollers contain 10-bit analog-to-digital converters (ADCs). The time taken by the ADC for one conversion cycle is determined by equation (5):

$$
T_{A D C}=\frac{1}{f_{A D C}} \cdot 14=14 / f_{A D C},
$$

where $f_{A D C}-\mathrm{ADC}$ clock frequency, $\mathrm{Hz}$;

14 - number of ADC clock periods required for one conversion [4].

The highest conversion accuracy is achieved, if $f_{A D C}$ is in the range of 50-200 kHz. With $f_{A D C}=100 \mathrm{kHz}$, the time of one conversion cycle will be $T_{A D C}=140 \mu \mathrm{s}$ in accordance with equation (5). 
Thus, the process of converting voltage to binary code using the ADC built into the microcontroller (MCU) is quite long and this ADC cannot be used to convert the instantaneous peak values $V_{s}$ max and $V_{s}$ mininto binary code.

\section{Materials and methods}

An experimental MCC sample implemented with the Arduino Uno is shown in Fig. 3. Arduino Uno is built on the ATmega328P MCU, which can communicate with a PC via the USB interface $[5 ; 6]$. MCC contains: two reference resistors $R_{o}$ and $R_{r}$; capacitive sensor $C_{s}$ and reference capacitor $C_{r}$; a RC low-pass filter consisting of a resistor $\mathrm{R}$ and a capacitor $\mathrm{C}$.

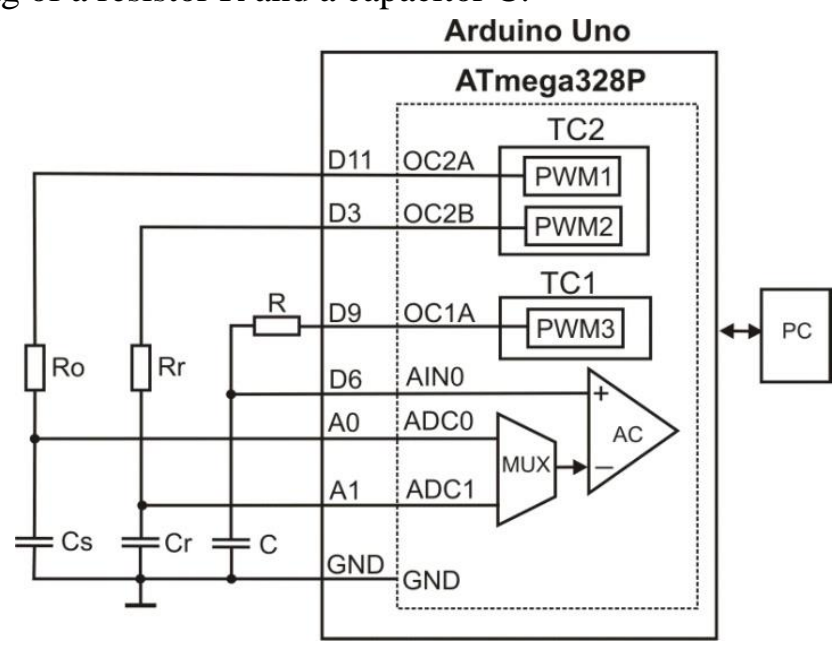

Fig. 3. Schematic diagram of MCC experimental sample

Pins A0, A1, D3, D6, D9 and D11 of the Arduino Uno (Fig. 3) are connected to the ATmega328P pins and have the following functional purpose:

- $\mathrm{ADC} 0$ and $\mathrm{ADC} 1$ - inputs of the analog multiplexer (MUX), the output of which is connected to the (-) input of the analog comparator (AC);

- OC2A and OC2B - outputs of pulse-width modulators (PWM1) and (PWM2), implemented on the basis of 8-bit timer/counter 2 (TC2);

- OC1A - PWM3 output implemented on the basis of 16-bit timer/counter 1 (TC1);

- $\quad$ AINO - input (+) AC.

The resistance $R_{o}$ was selected via the condition (6):

$$
R_{0} \cdot C_{\text {smin }} \geq 0.25 \cdot T_{P W M},
$$

where $\quad T_{P W M}=1 / f_{P W M}-$ period of signals generated at the PWM1 and PWM2 outputs, ms;

$C_{s \min }-$ minimum value of the $\mathrm{C}_{\mathrm{s}}$ sensor capacitance, $\mathrm{pF}$.

Values of reference circuit elements were selected via the condition (7):

$$
R_{r} \cdot C_{r}=R_{0} \cdot C_{s \text { min }} .
$$

Fig. 4 illustrates the changes in the values of $V_{r \min }, V_{r \max }, V_{s \min }$ and $V_{s \max }$ when $R_{r}=R_{o}, C_{r}=C_{s} \min$, $C_{s \max }=2 \cdot C_{s \min }$ and the condition (6) is fulfilled.

The high $V_{H}$ and low $V_{L}$ voltage levels in Fig. 4 correspond to logic "1" and " 0 " signals generated by PWM1 and PWM2 (Fig. 3). The study was carried out at a frequency $f_{P W M}=100 \mathrm{kHz}$, at $C_{s \min }=250 \mathrm{pF}, R_{o}=R_{r}=10 \mathrm{k} \Omega, C_{r}=C_{s \min }=250 \mathrm{pF}$, according to (6) and (7).

Algorithms for converting $V_{r}$ and $V_{s}$ into a binary code are the same, according to (2) and (3). Therefore, we will consider an algorithm for converting one of them, for example, $V_{r}$ into a binary code. The MCU executes the algorithm in the following order: 


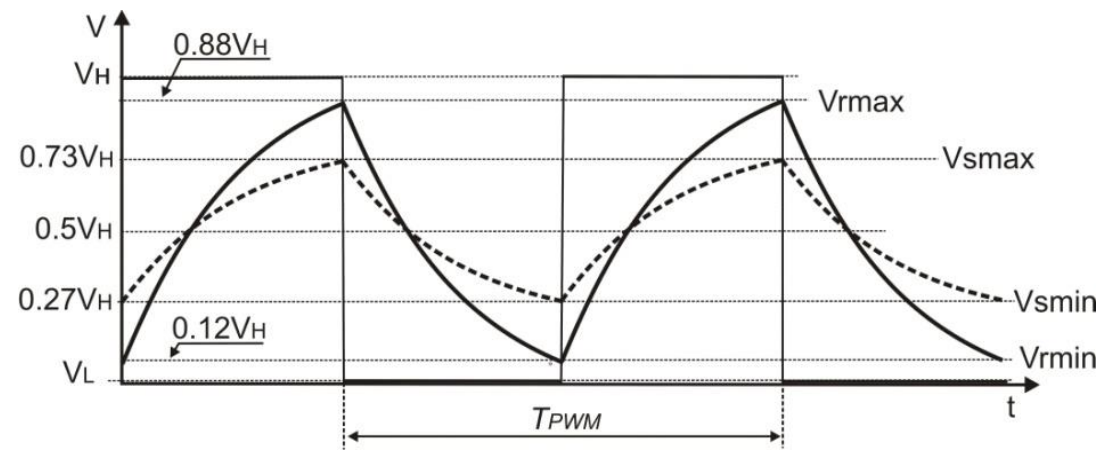

Fig. 4. Diagrams of voltage changes across Cs and Cr elements of MCC schema

Step 1. The MCU tunes PWM1 and PWM2 to a given frequency $f_{P W M}$ With duty cycle $G_{1}=G_{2}=0.5$ and starts PWM1 and PWM2, which operate synchronously.

Step 2. MCU connects ADC1 input (Fig. 3) of analog multiplexer (MUX) to the (-) input of the analog comparator (AC).

Step 3. MCU generates a signal at PWM3 output with a minimum fill factor $G_{3}$. This signal is applied to the RC filter input. The voltage generated at the output of the RC filter is determined by the expression: $V_{C}=G_{3} \cdot V_{H}$, where $V_{H} \approx 5 \mathrm{~V}$ is the high voltage level of the PWM3 signal, when the MCU is supplied with $5 \mathrm{~V}$.

For the case when $V_{C}<V_{r \text { min }}$ at the output of the AC there will be a logical "O".

Step 4. MCU starts to increase $G_{3}$. The $V_{C}$ voltage rises smoothly. As soon as $V_{C} \geq V_{r \min }$, then logic "1" appears at the output of the AC. MCU will store the binary code $N_{r \min }$ proportional to $G_{3}$. The stored binary code $N_{r \min }$ is equivalent to $V_{r \min }$.

Step 5. MCU generates a signal at the PWM3 output with the maximum $G_{3}$. If $V_{C}>V_{r \text { max }}$ at the output of the AC will be logic " 1 ".

Step 6. MCU starts decreasing $G_{3}$. The $V_{C}$ voltage at the output of the RC filter decreases smoothly. As soon as $V_{C} \leq V_{r \text { max }}$, then logic "O" will appear at thecomparator AC output. MCU will save the $N_{r \max }$ binary code proportional to $G_{3}$. The stored binary code $N_{r \max }$ is the equivalent of $V_{r \max }$.

Step 7. MCU calculates $N_{r}=N_{r \max }-N_{r \min }$. Binary code $N_{r}$ is equivalent to $V_{r}$.

Step 8. MCU connects the ADC0 input of the MUX to the (-) input of the comparator AC and performs a similar algorithm to determine $N_{s}=N_{s \max }-N_{s \min }$. Binary code $N_{r}$ is the equivalent of $V_{s}$.

Step 9. MCU determines $N_{r s}=N_{r}-N_{s}$ similarly to (1). Binary code $N_{r s}$ is equivalent to $V_{r s}$.

The PC (Fig. 3) receives the results of the conversions from MCU, performs their processing and displays the decimal equivalents of binary codes on the monitor (Fig. 5).

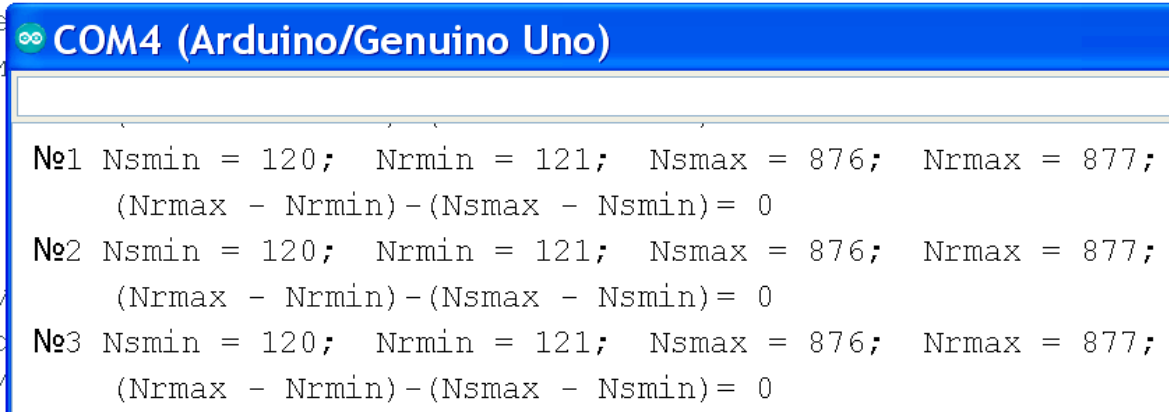

Fig. 5. Binary codes $N_{s \min }, N_{r \min }, N_{s \max }$ and $N_{r \max }$ on the PC monitor

\section{Results}

The results of experimental studies are shown in Tables 2 and 3. The values of $V_{\text {sminand }} V_{\text {smax }}$ are determined by the formulas (8):

$$
V_{s \min }=\frac{N_{s \min } \cdot V_{H}}{T O P} \text { and } V_{s \max }=\frac{N_{s \max } \cdot V_{H}}{T O P}
$$


where TOP - the PWM3 resolution.

For the considered case, TOP $=1000$. With $C_{s}=250 \mathrm{pF}, N_{s \min }=120\left(\right.$ Table 2) $V_{s \min }=120$. $5 / 1000=0.6 \mathrm{~V}$.

In our case, $\mathrm{TOP}=1000 . V_{s \min }=120 \cdot 5 / 1000=0.6 \mathrm{Vwith} C_{s}=250 \mathrm{pF}, N_{s \min }=120($ Table 2$)$.

Results of experimental studies MCC with $C_{r}=250 \mathrm{pF}$

Table 2

\begin{tabular}{|l|c|c|c|c|c|c|}
\hline $\boldsymbol{C}_{\boldsymbol{s}}, \mathbf{p F}$ & 250 & 300 & 350 & 400 & 450 & 500 \\
\hline $\boldsymbol{N}_{\boldsymbol{s m i n}}$ & 120 & 146 & 182 & 214 & 241 & 264 \\
\hline $\boldsymbol{N}_{\boldsymbol{s m a x}}$ & 876 & 849 & 813 & 781 & 754 & 731 \\
\hline $\boldsymbol{V}_{\boldsymbol{s m i n}}, \mathbf{V}$ & 0.6 & 0.73 & 0.91 & 1.07 & 1.205 & 1.32 \\
\hline $\boldsymbol{V}_{\boldsymbol{s m a x}}, \mathbf{V}$ & 4.38 & 4.245 & 4.065 & 3.905 & 3.77 & 3.655 \\
\hline $\boldsymbol{V}_{\boldsymbol{s}}=\boldsymbol{V}_{\boldsymbol{s m a x}}-\boldsymbol{V}_{\boldsymbol{s m i n}}, \mathbf{V}$ & 3.78 & 3.515 & 3.155 & 2.855 & 2.565 & 2.335 \\
\hline $\boldsymbol{N}_{\boldsymbol{r}}=\boldsymbol{N}_{\boldsymbol{r}}-\boldsymbol{N}_{\boldsymbol{s}}$ & 0 & 53 & 125 & 189 & 243 & 288 \\
\hline $\boldsymbol{V}_{\boldsymbol{r} \boldsymbol{s}}=\boldsymbol{V}_{\boldsymbol{r}}-\boldsymbol{V}_{\boldsymbol{s}}, \mathbf{V}$ & 0 & 0.265 & 0.625 & 0.945 & 1.215 & 1.44 \\
\hline
\end{tabular}

Table 3 shows the data measured with an oscilloscope connected to the $C_{s}$ sensor and the reference capacitor $C_{r}$ (the oscilloscope is not shown in Fig. 3).

Data measured with an oscilloscope connected to $C_{s}$ and $C_{r}=250 \mathrm{pF}$

Table 3

\begin{tabular}{|l|c|c|c|c|c|c|}
\hline $\boldsymbol{C}_{\boldsymbol{s}}, \mathbf{p F}$ & 250 & 300 & 350 & 400 & 450 & 500 \\
\hline $\boldsymbol{V}_{\boldsymbol{s m i n}}, \mathbf{V}$ & 0.59 & 0.69 & 0.84 & 1.00 & 1.16 & 1.28 \\
\hline $\boldsymbol{V}_{\text {smax }}, \mathbf{V}$ & 4.41 & 4.28 & 4.13 & 3.97 & 3.81 & 3.72 \\
\hline $\boldsymbol{V}_{\boldsymbol{s}}=\boldsymbol{V}_{\boldsymbol{s m a x}}-\boldsymbol{V}_{\text {smin }}, \mathbf{V}$ & 3,82 & 3.59 & 3.29 & 2.97 & 2.65 & 2.44 \\
\hline $\boldsymbol{V}_{\boldsymbol{r}}=\boldsymbol{V}_{\boldsymbol{r}}-\boldsymbol{V}_{\boldsymbol{s}}, \mathbf{V}$ & 0 & 0.23 & 0.53 & 0.85 & 1.17 & 1.38 \\
\hline
\end{tabular}

Oscillograms of voltages across $C_{s}$ and $C_{r}$ are shown in Fig. 6. In the experiments, the capacitance of the connecting probes of the oscilloscope was taken into account. Fig. 7 shows three dependences of $V_{r s}$ on $C_{s}$, at $C_{r}=250 \mathrm{pF}$, obtained according to (1). Dependence 1 - simulation in Multisim, Dependence 2 - experimental studies, Dependence 3 - oscilloscope measurements of voltages across $C_{s}$ and $C_{r}$. The dependences were obtained at $V_{H}=5 \mathrm{~V}$.

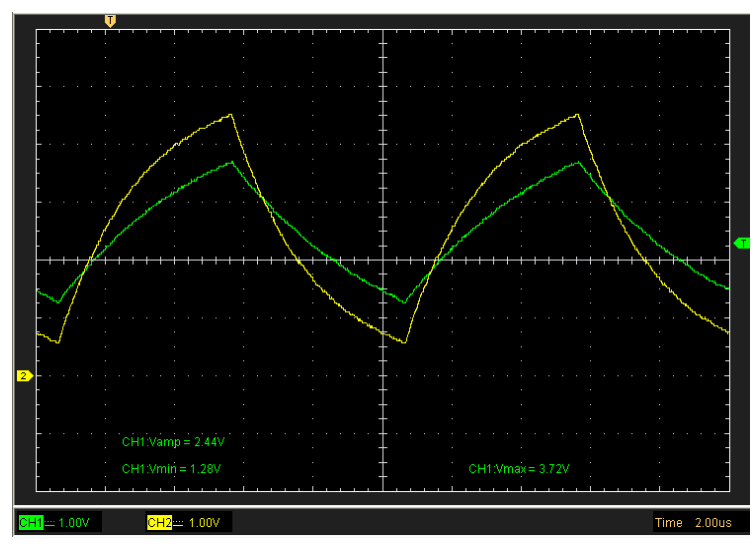

Fig. 6. Oscillograms of voltages across $\mathrm{C}_{s}(\mathrm{CH} 1)$ and across $C_{r}(\mathrm{CH} 2)$

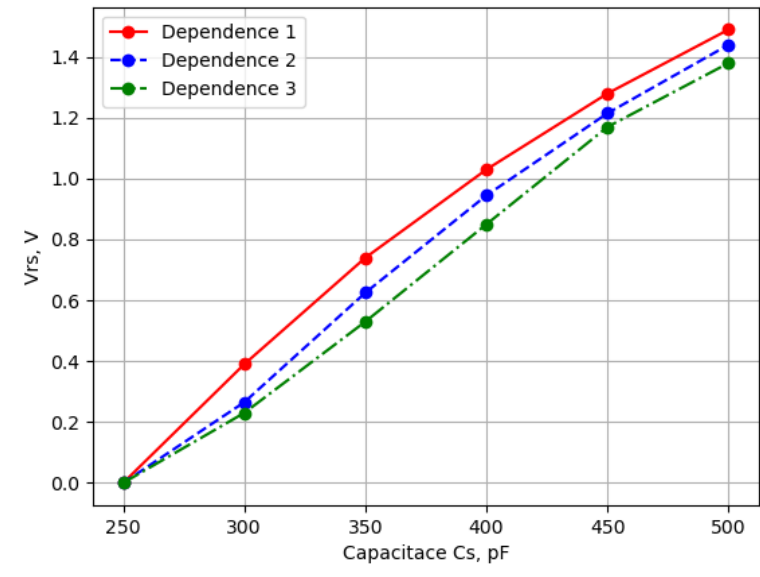

Fig. 7. Dependences of $V_{r s}, V$ on $C_{s}$

The benefit of the proposed MCC in comparison with the closest analogues $[7 ; 8]$ is that its implementation is completed without external additional functional elements on a single microcontroller. This circumstance expands the areas of MCC using and increases the possibilities for its improvement by changing only the software without changing the hardware.

MCU allows us to change the frequency and fill factor of the PWM1 and PWM2 signals. Management of the measuring signal parameters in the implementation of multi-parameters moisture 
meters increases the measurement accuracy $[9 ; 10]$. The frequency and fill factor control function is not available in the known MCCs.

\section{Conclusions}

1. The dependences of the output signal on the sensor capacitance are obtained, confirming the adequacy of the MCC model to the designed device.

2. $V_{r \max }=0.88 V_{H}$ and $V_{r \min }=0.12 V_{H}$ necessary for designing of the optimal MCC are obtained.

3. The results of the work allow us to design MCC depending on the required frequency of PWM1 and PWM2, resolution and initial capacitance of the sensor.

4. The MCC under study can be built on ATmega32U4 that contains a USART/USB interface converter and will reduce the prime cost of the USB-moisture meter.

5. The MCC algorithm and program can be used in the educational process to study microprocessorbased measuring devices.

\section{References}

[1] Jafari M., Chegini G.R., Rezaeealam B, Shaygani Akmal A.A. Experimental determination of the dielectric constant of wheat grain and cluster straw in different moisture contents. Food Sci Nutr. 2019;00:1-7. [online] [20.03.2021].Available at: https ://doi.org/10.1002/fsn3.1350.

[2] Цымбал А.А., Будников Д.А. Диэлектрические свойства зерновых (Dielectric properties of grain) // Bulletin of VNIIMZh. 2015. No. 4, pp. 52-55. (In Russian). [online] [19.03.2021]. Available at: http://www.vniimzh.ru/images/material/Magazines/n24.pdf.

[3] Вострухин А.В., Вахтина Е.А., Болдырев И.А. Пат. № 2698492 РФ на изобретение. Микроконтроллерное измерительное устройство емкости для встраиваемых вычислительных систем контроля и управления. (Pat. 2698492. Microcontroller capacitance measuring device for embedded computing systems of control and management) Stavropol State Agrarian University, Stavropol, Russia,2019, bulletin No 25. (In Russian). [online] [30.04.2020]. Available at: https://patents.s3.yandex.net/RU2698492C1_20190828.pdf

[4] ATmega328/P. Datasheet complete. [online] [24.03.2021]. Available at: http://mkprog.ru/wpcontent/uploads/2017/09/ATmega328-328P_Datasheet.pdf.

[5] Blum J. Exploring Arduino: Tools and Techniques for Engineering Wizardry. Wiley, 2013. 384 p.

[6] Yoder R. B. An Arduino-Based Alternative to the Traditional Electronics Laboratory. 2015 BFY Proceedings. July 22-24, 2015,Published by the American Association of Physics Teachers, pp. 107-110. [online][22.02.2021]. Available at: https://advlabs.aapt.org/items/detail.cfm?ID = 13820 .

[7] Assaad M., Zubair A.M.,Tang T.B.A semi-cylindrical capacitive-based differential sensing system for water content in crude oil measurement. International Review on Modeling and Simulations. 7,2014, pp. 213-220. [online] [22.03.2021]. Available at: https://www.researchgate.net/publication/288064419_A_semi-cylindrical_capacitivebased_differential_sensing_system_for_water_content_in_crude_oil_measurement.

[8] Vostrukhin A., Vakhtina E., MastepanenkoM., Gabrielyan Sh. Dielectric USB-Moisture Meter for seeds of agricultural crops. Proceedings of 8th International Scientific Conference "Rural Development 2017”, November 23-24, 2017 Aleksandras Stulginskis University, Lithuania,[online] [26.03.2021]. Available at: http://conf.rd.asu.lt/index.php/rd/article/view/407/

[9] Дубров Н.С., Кричевский Е.С., Невзлин Б.И.. Многопараметрические влагомеры для сыпучих материалов (Multi-parameter moisture meters for bulk materials). Moscow: Mechanical Engineering, 1980. 144 p. (In Russian).

[10] Dielcometric moisture meter FIZEPR-SW100.30 for laboratory research. [online] [21.03.2021]. Available at: https://fizepr.com/laboratory-dielectric-moisture-meter. 